\title{
Análise de Tecnologias de Informação e Comunicação para Apoio à Tradução para a Língua Brasileira de Sinais no Ambiente Educacional
}

\author{
Rodrigo Araújo ${ }^{1}$, Simara Rocha $^{1}$, Aurea Melo ${ }^{2}$, \\ Ana Oran ${ }^{3}$, Davi Viana ${ }^{4}$, Luis Rivero ${ }^{4}$ \\ ${ }^{1}$ Departamento de Informática - UFMA \\ São Luis - MA, Brasil \\ ${ }^{2}$ Universidade Estadual do Amazonas - UEA \\ Amazonas - AM, Brasil \\ ${ }^{3}$ Universidade Federal do Amazonas - UFAM \\ Amazonas - AM, Brasil \\ ${ }^{4}$ Programa de Pós-Graduação em Informática - UFMA \\ São Luis - MA, Brasil

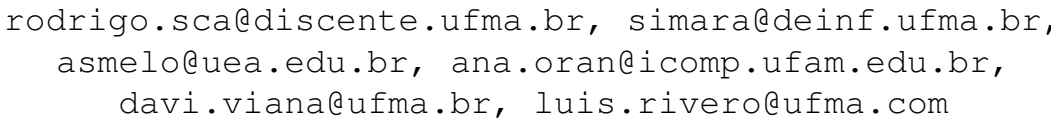

\begin{abstract}
In deaf education, it is important to use visual tools and methodologies in the teaching process. However, regarding the information and communication technologies to support the translation of sentences into Brazilian Sign Language (LIBRAS), there is no consensus on which technology to use. This paper presents an analysis of the existing tools for this purpose. A survey and analysis were made of: (a) the functionalities of the applications; $(b)$ the evaluations of the users of the applications studied; and (c) interviews with professionals about their experiences with the use of these applications in the educational environment. As a result, it is possible to understand the potential of these tools in the process of teaching the deaf and to realize which is the most promising tool.
\end{abstract}

Resumo. Na educação de surdos é importante a utilização de ferramentas e metodologias visuais no processo de ensino. Porém, quanto às tecnologias de informação e comunicação para apoio à tradução de sentenças para a Língua Brasileira de Sinais (LIBRAS), não há consenso sobre qual tecnologia utilizar. Este artigo apresenta uma análise das ferramentas existentes para este fim. Foi feito o levantamento e análise: (a) das funcionalidades dos aplicativos; (b) das avaliações dos usuários dos aplicativos estudados; $e(c)$ de entrevistas com profissionais sobre suas experiências com o uso desses aplicativos no ambiente educacional. Como resultado, pode-se entender o potencial dessas ferramentas no processo de ensino a surdos e perceber qual ferramenta é a mais promissora.

\section{Introdução}

No processo de educação de pessoas surdas é de grande importância a utilização do elemento visual como norteador para ferramentas e metodologias de ensino, pois estes 
são elementos facilitadores do desenvolvimento do aprendizado desse tipo de público Almeida et al. 2007]. A visualidade, para o surdo, representa o principal canal de processamento de experiências, pois pode propiciar a aquisição, construção e expressão de valores, vivências e conhecimento que de outra maneira não seriam comunicáveis [Pinto et al. 2012]. Portanto, as metodologias que são utilizadas para a educação de pessoas surdas precisam priorizar recursos visuais para facilitar o entendimento e a comunicação entre alunos e professores [Almeida et al. 2007].

Uma das ferramentas disponíveis para uso dentro do processo de ensino de surdos é o tradutor para a LIBRAS. Essa ferramenta converte textos, imagens de textos ou áudios para a língua de sinais, que é representada através de um avatar animado [Corrêa et al. 2014a]. Ela também favorece a socialização e auxilia na interação entre surdos e ouvintes, amenizando, inclusive, processos de segregação social e auxiliando na promoção de experiências de escolarização inclusivas [Corrêa et al. 2014a].

Apesar da existência de trabalhos relatando o uso destas tecnologias no ambiente educacional de pessoas surdas [Corrêa et al. 2018, Bandeira et al. 2018, Silva 2021], poucos são estudos comparativos das ferramentas existentes para este fim. Diante do exposto, este artigo tem como objetivo a análise de tradutores para Língua Brasileira de Sinais (LIBRAS) considerando as suas funcionalidades e a opinião de possíveis usuários. Este trabalho considerou os seguintes aplicativos móveis nessa categoria: Hand Talk, Giulia, VLibras, Rybená, Libras Lens e Acesso Brasil.

O restante deste trabalho está organizado como segue. A Seção 2 apresenta trabalhos sobre o uso de tecnologias de informação e comunicação na aplicação de LIBRAS. A Seção 3 apresenta a metodologia utilizada, a Seção 4, os resultados das análises e a discussão e, finalmente, a Seção 5 apresenta as conclusões e trabalhos futuros decorrentes dos resultados deste artigo.

\section{Tecnologias de Informação e Comunicação na Aplicação de LIBRAS}

A seguir, são apresentados trabalhos que analisam o uso de tecnologias de informação e comunicação na aplicação de LIBRAS e seu impacto na comunicação e educação. Estes trabalhos serão apresentados seguindo a ordem cronológica de publicação, do mais antigo ao mais recente.

Dentre os trabalhos sobre o uso de aplicativos de tradução para LIBRAS na Educação, podemos citar Corrêa et al. (2014) [Corrêa et al. 2014b], que fala, em seu trabalho, sobre a inserção de aplicativos de tradução para promover uma melhor comunicação entre surdos e ouvintes, usando o ProDeaf e o Hand Talk. Como resultado, os autores relatam que o ambos os aplicativos são capazes de contribuir para inclusão social e para a comunicação, desde que salvaguardados três elementos: (a) a posse de um smartphone compatível com os aplicativos; (b) acesso a internet móvel; e (c) o conhecimento sobre como utilizá-los.

Também pode ser citado o trabalho de Bandeira et al. (2018), onde os autores relatam o uso de aplicativos de tradução para LIBRAS na educação para surdos. No trabalho, os autores investigaram o uso do aplicativo Hand Talk do ponto de vista dos docentes em prol do benefício dos alunos surdos dentro do ambiente educacional. Como resultado, os autores concluíram que a utilização do aplicativo Hand Talk pode ser considerada como 
proveitosa e funcional. Entretanto, constataram que apenas o aplicativo não era suficiente para um aprendizado ideal, tendo em vista que a tradução não leva em conta regionalismos, provocando, em certas situações, dificuldade no aprendizado.

No trabalho de Corrêa et al. (2018), os autores falam sobre o uso de aplicativos na educação bilíngue de pessoas surdas. Os autores realizaram uma pesquisa com a inserção de dois aplicativos na educação para surdos, o Hand Talk e o ProDeaf. Os autores identificaram, nos resultados, maior incidência de datilologia (soletração manual do alfabeto da Língua Portuguesa) no aplicativo ProDeaf, implicando em um maior vocabulário no Hand Talk. Além da necessidade de aumento do vocabulário dentro dos aplicativos para uma melhor efetividade na comunicação.

Além dos trabalhos citados, outros podem ser considerados. Oliveira et al. (2019) trata em seu artigo sobre a inclusão do aplicativo Hand Talk como ferramenta de apoio a professores de ciências na educação inclusiva. Como resultado, o autor indicou que a utilização desses recursos em contextos educacionais promove a inclusão digital de alunos com surdez. Ele também constata que o Hand Talk é uma importante ferramenta a ser inserida no ambiente educacional, pois traz a possibilidade de melhoria da comunicação entre surdos e ouvintes e um salto qualitativo do processo de ensino e aprendizagem.

Por sua vez, o trabalho de Silva (2021), apresenta uma análise comparativa entre os aplicativos de tradução para libras, Hand Talk e VLibras. Como resultado, o autor identificou que a principal funcionalidade dos dois, a tradução, é disponibilizada de maneira igualitária, porém o dicionário do VLibras, apesar de não estruturado por categorias como o do Hand Talk, propicia ao usuário uma observação mais precisa de palavras com a mesma estrutura e significados diferentes. $\mathrm{O}$ autor também constatou a relevância social desses recursos, como uma alternativa de promoção da acessibilidade para surdos.

De um modo geral, os estudos sobre o uso de ferramentas tecnológicas na educação e comunicação dos surdos têm crescido nos últimos anos. Entretanto, os relatos dos autores citados não possuem uma análise comparativa ampla dos vários aplicativos de tradução que estão disponíveis atualmente no mercado e suas funcionalidades. Além disso, não são consideradas as avaliações dos usuários nas plataformas que disponibilizam esses aplicativos. O relato dessas análises e avaliações comparativas pode ser importante para que participantes do processo de ensino e aprendizagem escolham qual aplicativo utilizar e para que desenvolvedores possam entender os pontos e funcionalidades que eles podem ou não inserir em seus projetos. Nesse sentido, a seção a seguir apresenta a metodologia adotada para alcançar este objetivo.

\section{Método}

Este trabalho analisou três fontes de dados: (a) aplicativos de tradução para LIBRAS para Android disponíveis no mercado; (b) avaliação dos usuários dos aplicativos selecionados na plataforma Google PlayStore; e (c) entrevistas com profissionais da educação de surdos, relatando suas experiências com o uso deste tipo de ferramenta.

Quanto ao levantamento dos aplicativos disponíveis no mercado para tradução para LIBRAS, foi utilizada a metodologia adaptada de Gil (2008). Foi aplicada a string "LIBRAS”AND "Linguagem de Sinais Brasileira”AND "Linguagem Brasileira de Sinais"AND "Surdo"AND "Tradutor Libras", permitindo identificar seis aplicativos relacionados (ordenados por média de avaliação): Hand Talk, Giulia, VLibras, Rybená, 
Libras Lens, e Acesso Brasil. As strings foram escolhidas para identificar quaisquer aplicativos no contexto de surdos, evitando correr o risco de não considerar algum aplicativo relacionado com este trabalho. Embora houvesse aplicativos relacionados a LIBRAS em outros contextos ( Bíblia em LIBRAS, dicionários, entre outros), apenas aplicativos com a função de tradutor foram selecionados. Ademais, outros critérios foram definidos: (a) Aplicativos Mobile de acesso gratuito e (b) Aplicativos Mobile que funcionam no sistema operacional Android, em qualquer versão. O sistema operacional Android foi selecionado como objeto alvo deste trabalho, considerando que dispositivos com sistema operacional Android predominam no mercado [Lima 2017]. A seguir são apresentados os aplicativos identificados.

(a) Hand Talk: O Hand Talk, desenvolvido pela empresa Hand Talk, é descrito como um aplicativo de tradução e aprendizado de sinais em LIBRAS e ASL (American Signal Language). Ele traduz áudio e texto para a Língua de Sinal, que é reproduzida através de um personagem animado. Além disso, o Hand Talk possui um dicionário de termos e palavras e vídeos aulas. Ele foi eleito pela ONU o melhor app Social do mundo [Terenzi 2017]. A versão utilizada do aplicativo foi a versão 3.3.3.

(b) Giulia: Giulia é um tradutor e plataforma de comunicação para surdos. Seu objetivo é permitir que surdos possam ser compreendidos por ouvintes que não possuem conhecimento de LIBRAS e que ouvintes possam se comunicar com surdos sem ter o conhecimento. Para a representação, ele utiliza imagens e um personagem animado em 3D. Desenvolvido pela Map Innovation. A versão utilizada do aplicativo foi a versão 2.1.2.

(c) VLibras: O VLibras é descrito como um aplicativo de tradução do Português para Libras. Ele utiliza a representação de personagem animado em 3D para as suas traduções. O VLibras foi desenvolvido pelo Laboratório de Aplicações de Vídeo Digital (LAViD), da UFPB (Universidade Federal da Paraíba), com o apoio do Ministério do Planejamento, Orçamento e Gestão (MPOG), Secretaria de Direitos Humanos (SHD/PR) e da Rede Nacional de Ensino e Pesquisa (RNP). A versão utilizada do VLibras foi 3.3.2.

(d) Rybená Tradutor Libras Voz (Rybená) O Rybená é um aplicativo que transforma voz em textos escritos, texto escrito em voz e traduz textos para LIBRAS. A representação da tradução para LIBRAS é feita através de um personagem 3D. Desenvolvido pelo Grupo ICTS. A versão utilizada foi a 3.0.13.

(e) Libras Lens O Libras Lens é um aplicativo que traduz textos em Português para LIBRAS usando a câmera do dispositivo para identificar os textos do mundo real e traduzi-los para LIBRAS. Ele utiliza a representação de um personagem animado em 3D. Ele foi desenvolvido por Devnicius. A versão do Libras Lens utilizada foi a 1.0.1.

(f) Acesso Brasil: Desenvolvido pela MDS Tecnologia da Informação Ltda., o Acesso Brasil transforma texto em português para versões na Língua Brasileira 
de Sinais, podendo também traduzir textos orais, utilizando um personagem animado em 3D que representa a sentença em LIBRAS. A versão utilizada do Acesso Brasil foi a 1.1.0.

Para a análise da primeira fase, foram levantadas as principais funcionalidades de cada aplicativo e depois comparadas com as funcionalidades dos outros aplicativos. A classificação dessas funcionalidades foi dividida em três categorias: (a) A (Atende); (b) AP (Atende Parcialmente); e (c) NA (Não Atende).

$\mathrm{Na}$ análise, receberam categoria $\mathrm{A}$ na lista de funcionalidades os aplicativos que: (i) Possuem a funcionalidade; (ii) A funcionalidade do aplicativo atende corretamente o proposto por ela, com dados e conteúdo relevantes, sem nenhuma dificuldade para executá-la; e (iii) a funcionalidade possui poucos, ou nenhum, erros em sua execução e, caso possua erros, nenhum erro que comprometa a execução correta da funcionalidade. A categoria AP recebem os aplicativos que: (i) Possuem a funcionalidade; (ii) A funcionalidade atende parcialmente o proposto por ela, com dados e conteúdos relevantes, além de pouca ou nenhuma dificuldade para executá-la; e (iii) A funcionalidade possui poucos, ou nenhum, erros em sua execução e, caso possua erros, nenhum erro que comprometa a execução correta da funcionalidade. Por fim, recebem a categoria NA na lista de funcionalidades os aplicativos que não possuem ou não executam a funcionalidade.

Na segunda fase foi feita a análise dos comentários e avaliações dos usuários desses aplicativos. O resultado dessa fase foi compilado de duas formas: A primeira delas sendo a média de avaliação por estrelas, com o máximo de 5 estrelas e o mínimo de 1 estrela, dadas pelos próprios usuários. A segunda fase, os pontos positivos e negativos de cada aplicativo, com base nas avaliações descritivas dos usuários. Os comentários selecionados falam sobre funcionalidades, erros, desempenho ou da experiência de uso.

A terceira consistiu na entrevista com profissionais que atuam ou atuaram na educação de surdos, com experiência no uso de aplicativos tradutores para LIBRAS dentro do ambiente educacional. Os relatos solicitados na entrevista foram de situações onde os aplicativos tradutores para LIBRAS, foram ou poderiam ter sido úteis dentro do ambiente educacional e como as funcionalidades dos aplicativos contribuíram no processo de ensino. Foram entrevistados cinco professores, um homem e quatro mulheres. Dentre os entrevistados, um atua na rede pública de ensino, três na rede particular e um já atuou na rede pública de ensino. Quatro deles atuaram como professores e intérpretes e um atuou como professor auxiliar. A seção a seguir, apresenta os resultados obtidos na análise.

\section{Resultados e Discussão}

\subsection{Análise de Características e Funcionalidades}

O Quadro 1 reúne informações da análise de funcionalidades dos 6 aplicativos escolhidos. Após a análise dos 6 aplicativos, evidenciaremos os seus diferenciais e comparação obtidos, quanto às suas características e funcionalidades.

O aplicativo Hand Talk, possui o maior conjunto de funcionalidades, sendo, com base na análise de funcionalidades, o mais efetivo no serviço de tradução para LIBRAS, uma vez que praticamente todas as funcionalidades por ele apresentadas se enquadram na categoria A (Atendida), com exceção a disponibilidade offline. Como diferenciais em suas funcionalidades, podemos destacar que ele funciona offline, apesar de que, em 
seu módulo offline, ele utiliza-se apenas de datilologia para a tradução, por esse motivo enquadrando-se na categoria AP. A sincronia com as redes sociais e o sistema de login tornam a melhor a experiência, uma vez que o usuário pode manter salvo seu histórico de tradução, sua progressão nas vídeo aulas, e suas palavras salvas no dicionário.

O Aplicativo Giulia possui um conjunto satisfatório de funcionalidades e se enquadra na categoria A em praticamente todas as suas funcionalidades apresentadas. Ele também funciona offline, utilizando a datilologia para a tradução quando não conectado à rede. Um ponto importante a ser ressaltado é que sua funcionalidade de Cadastro e Login, apesar de trazer diversos benefícios, como salvar o progresso nas videoaulas e favoritar termos e traduções, é obrigatória e, por esse fato, foi classificada como AP (Atende Parcialmente), pois, por ser obrigatória, pode consistir em uma desvantagem. Outro critério para a disponibilidade offline também ser considerada como AP foi que, no primeiro acesso, para utilizar as funcionalidades do aplicativo, é necessário fazer o cadastro e login e, para isso, é necessário estar conectado. Com exceção da questão do Cadastro e Login, o funcionamento offline do aplicativo se dá de forma adequada, semelhante ao Hand Talk.

Quadro 1 - Análise de Funcionalidades

\begin{tabular}{|l|l|l|l|l|l|l|}
\hline Funcionalidades & Hand Talk & Giulia & VLibras & Rybená & Libra Lens & Acesso Brasil \\
\hline Transcrição de Texto para Língua de Sinais & A & A & A & A & A & AP \\
\hline Transcrição de Áudio para Língua de Sinais & A & A & A & A & NA & AP \\
\hline Dicionário & A & AP & AP & NA & NA & NA \\
\hline Videoaulas & A & A & NA & NA & NA & NA \\
\hline Sincronia com as Redes Sociais & A & A & NA & NA & NA & NA \\
\hline Ajuda & A & AP & NA & NA & NA & AP \\
\hline Personalização de Avatar & A & NA & NA & NA & NA & NA \\
\hline Disponibilidade Offline & AP & AP & NA & NA & NA & NA \\
\hline Cadastro e Login & A & AP & NA & NA & NA & NA \\
\hline Transcrição de Áudio para Texto & A & A & AP & A & A & AP \\
\hline Regulador de Velocidade dos sinais & A & NA & A & A & NA & NA \\
\hline Compartilhamento de Tradução & NA & NA & A A & A & A & NA \\
\hline Código aberto (Open Source) & NA & NA & A & NA & NA & NA \\
\hline Regionalismo & NA & NA & NA & NA & A \\
\hline Captura de Texto via Camera & & & NA \\
\hline
\end{tabular}

O aplicativo VLibras possui um diferencial com relação aos outros, que é o Regionalismo. Com essa funcionalidade é possível escolher o Estado Brasileiro e a variação linguística de sua preferência, o que pode tornar o entendimento melhor pelo usuário [Bandeira et al. 2018]. A língua manifesta as mudanças que ocorrem decorrentes da influência do ambiente físico e sociocultural [Isquerdo 1996], portanto esse é um fator diferencial importante. Além disso, o VLibras é um aplicativo de código aberto, possibilitando o uso do seu código fonte para estudos e criações de novas ferramentas.

Com relação ao aplicativo Rybená, foi constatado que todas as funcionalidades oferecidas por ele são categoria A, entretanto ele oferece apenas cinco funcionalidades. Outra característica do Rybená é que ele solicita permissão para acesso a dados privados contidos no dispositivo, como acesso a fotos, dados pessoais e atividade do celular, o que pode ser considerado um ponto negativo.

O Libras Lens, por sua vez, também entrega todas as funcionalidades que ele possui com categoria A, mas o seu principal ponto positivo é a funcionalidade de Captura de texto via Câmera, uma funcionalidade não encontrada em nenhum dos outros aplicativos. 
Entretanto, outras funcionalidades importantes como a tradução de áudio para a língua de sinais, presente em todos os outros, ele não possui. O Libras Lens é um software de código aberto que utiliza o plug-in do VLibras para a tradução de suas capturas.

O último aplicativo analisado, Acesso Brasil, possui o menor conjunto de funcionalidades dentre os aplicativos analisados e todas são entregues com categoria AP.

Quanto a algumas funcionalidades e características em particular, a Personalização de Avatar foi considerada dentro desse contexto, uma vez que, dentro do ambiente educacional, os personagens 3D melhoram e ampliam a efetividade das aplicações de educação e treinamento [Rodrigues et al. 2012]. A Sincronia com as Redes Sociais e Cadastro e Login não foram considerados como uma mesma funcionalidade, uma vez que a Sincronia com as Redes Sociais oferece outras funcionalidades, como o convite de amigos para o uso do aplicativo e o compartilhamento na rede social escolhida, em específico, de forma facilitada. O Código aberto também é importante ser considerado, uma vez que um aplicativo que possui o código aberto permite que, a partir dele, outros aplicativos possam ser desenvolvidos e, no futuro, possa haver uma quantidade maior de opções para uso.

\subsection{Análise das Avaliações dos Usuários}

A seguir estão descritas para cada aplicativo: (a) Média de Avaliação dos usuários, dadas através de estrelas, sendo 5 estrelas a avaliação máxima e 1 estrela a avaliação mínima; (b) Pontos positivos e negativos com base nos comentários dos usuários; e (c) alguns comentários feitos pelos usuários.

(a) Hand Talk: O Hand Talk possui a média de avaliação de 4.4 estrelas. Ele tem como pontos positivos a grande quantidade de funcionalidades, ser intuitivo, fácil de usar, ter videoaulas de qualidade e ser leve, funcionando na maioria dos celulares. Ele ainda possui a opção de tradução para American Signal Language. Os pontos negativos destacados pelos usuários em suas avaliações, foram os erros de tradução ocasionais, o excesso no uso de datilologia, problemas no login e propagandas em excesso. Alguns dos comentários de usuários sobre o aplicativo estão descritos a seguir: "O app é muito bom, mas está travando muito. $O$ excesso de propagandas é demais e atrapalha com cliques acidentais"; " $O$ app está apresentando falhas. Se coloco uma palavra para ser sinalizada, ele não a realiza, apenas faz a datilologia”; "Amei o aplicativo, muito fácil de mexer."; "Excelente aplicativo, nunca vi nada igual!!!"; "Muito bom mesmo, acredito que não tem nenhum outro aplicativo desse tipo que seja melhor que o Hand Talk”

(b) Giulia: A média de avaliação do Giulia é 4.1 estrelas. O aplicativo tem como pontos positivos destacados a sua praticidade para realizar as traduções, acessibilidade e a facilidade de uso. Como pontos negativos descrito pelos usuários estão destacados os fechamentos inesperados do aplicativo, a solicitação de dados pessoais do usuário (como o número da identidade), o travamento na reprodução das traduções e algumas traduções erradas. Alguns dos comentários de de usuários sobre o aplicativo estão descritos a seguir: "Muito fácil e prático para realizar traduções"; "Fácil uso, muito intuitivo"; "Algumas traduções estão erradas e possui muita soletração."; "O aplicativo trava muito e demora para 
carregar as traduções”;

(c) VLibras: O VLibras possui a média de avaliação de 4.0 estrelas. Como pontos positivos, ele possui a clareza na reprodução dos sinais, a opção de controle de velocidade, ser intuitivo, fácil de usar e leve. Como pontos negativos, foram destacados alguns erros de tradução, erros no Regionalismo e uso ocasional de datilologia para um sinal já existente na língua. Alguns dos comentários de usuários sobre o aplicativo estão descritos a seguir: "Aplicativo muito bom, mostra com clareza os movimentos, tendo a opção de velocidade e fala."; "Poderia aceitar o incremento no vocabulário, pois recorre com frequência a soletração"; "A opção de regionalismo nem sempre funciona, precisa ser corrigida."; "Os movimentos do boneco não são fluidos, dificulta para entender";

(d) Rybená: O Rybená possui uma avaliação de 3.7 estrelas. Com pontos positivos são destacados pelos usuários o reconhecimento funcional de palavras e sentenças, a facilidade do uso, as opções de velocidade de tradução, ser fácil de entender e intuitivo. Os pontos negativos principais foram as falhas na tradução, desempenho ruim do aplicativo, fechamentos de forma inesperada e a exigência de acesso a dados pessoais dos usuários. Alguns dos comentários de usuários sobre o aplicativo estão descritos a seguir: "Além de traduzir para os surdos ele ensina a se comunicar em libras. Muito fácil e intuitivo de usar. Recomendo.”; "Tem a opção de definir a velocidade da fala em LIBRAS, muito bom"; "Fácil de usar e entender!"; "Não entendi muito bem a necessidade do app pedir dados privados. Não uso mais por causa disso."; "muitas falhas de tradução"; "sou surdo...mas gosto de app Rybena tradutor libras. Eu preciso ler juntos tradutor sinal conhece palavra. Fácil usar. Muita função". Sendo o último comentário descrito o comentário de um usuário surdo.

(e) Libras Lens: O Libras Lens possui uma média 3.0 estrelas em sua avaliação. Como ponto positivo, foi destacado a facilidade do uso e o seu grande destaque que é a tradução captura de textos do mundo real com a câmera do celular. Como ponto negativo, a demora para carregar a tradução e a falta da funcionalidade de traduzir áudio para LIBRAS. Alguns dos comentários de usuários sobre o aplicativo estão descritos a seguir: A base dos comentários foi: "Muito ruim a demora para carregar o texto."; "Fácil de usar para surdos e ouvintes."; "A função de extrair tradução de imagens de texto é muito útil e bastante funcional."

(f) Acesso Brasil: Esse aplicativo possui a avaliação mais baixa dentre todos os outros, sendo 2.9 estrelas. Como ponto positivo foi destacado a sua facilidade de uso. Como pontos negativos, se destacam a baixa qualidade das animações das traduções, os erros nas traduções, os travamentos, e o fechamento inesperado do aplicativo. Alguns dos comentários dos usuários foram: "Não funciona! Vive travado e não interpreta coisa alguma!"; "Gráficos ruins, não dá para compreender o que fala. Uma pena!"; "Parabéns fácil usar. Sou surda libras". Esse último comentário sendo de uma usuária surda.

A seguir estão descritas informações com base nos relatos de profissionais da 
educação a surdos.

\subsection{Entrevista com Profissionais que Trabalham com Surdos}

O relato subjetivo das experiências de profissionais da educação de surdos descreveu, em partes, como o uso das tecnologias de informação e comunicação podem ser úteis quando aplicados no ensino de surdos. Com base neles, foi possível identificar que o principal ponto de melhoria com o uso dessas tecnologias é a comunicação entre alunos, colegas e professores. Outra melhoria identificada pelos relatos é a compreensão de alguns termos pelos surdos, devido a utilização do recurso visual.

Em uma das entrevistas, uma das professoras destacou uma situação onde ela observou a estratégia de uma de suas alunas surdas, da rede pública municipal de ensino, para entender o que era conversado ao redor dela pelos seus colegas e familiares. $\mathrm{Na}$ situação, a aluna utilizava-se do recurso de captura de áudio do Hand Talk para traduzir para LIBRAS as conversas ao seu redor e, assim, compreender o que estava sendo dito. Com base nessa experiência, a professora pode entender melhor de que forma esses aplicativos auxiliam na comunicação entre surdos e ouvintes, além de auxiliar na compreensão do surdo das informações do mundo ao seu redor. Com isso, surdos e ouvintes podem romper barreiras de comunicação com o uso dos aplicativos [Corrêa et al. 2017].

Um professor da rede privada de ensino relatou que em uma turma de surdos havia um aluno que tinha muita dificuldade em aprender a ler frases e textos. Porém, com o uso do aplicativo Libras Lens, o professor pôde proporcionar ao aluno a experiência de ter o texto escrito traduzido para LIBRAS, através da funcionalidade de tradução da captura de textos do mundo real com a câmera do celular. De acordo com o relato, isso foi essencial para o desenvolvimento do aluno no decorrer do período letivo.

Outra professora relata, com base em suas experiências, que o uso do aplicativo Hand Talk no ambiente de ensino auxilia bastante no aprendizado do aluno, principalmente com a associação visual de uma palavra escrita a um sinal de libras, representados no aplicativo pelo personagem 3D, o Hugo. Ela, entretanto, também relata que uma das problemáticas enfrentadas com o uso do aplicativo é a diferença de sinais em diferentes regiões. De acordo com ela, a depender do Estado Brasileiro, há grandes diferenças entre os sinais ensinados e é um pouco difícil o uso dos aplicativos quando há essas situações, pois, algumas vezes, para alguns sinais específicos, os aplicativos traduzem utilizando os sinais do local onde a maior parte da equipe desenvolvimento se concentra, então os sinais ensinados em uma sala de aula de outra região, ou Estado, são diferentes dos sinais aplicativo, para uma mesma palavra, e isso causa confusão em alguns alunos.

Os relatos obtidos das entrevistas ressaltam como o uso de ferramentas tecnológicas, como os aplicativos tradutores para LIBRAS, foram relevantes dentro do ambiente educacional. De acordo com os relatos, na maioria das situações dentro do ambiente escolar eles utilizaram o Hand Talk que, de acordo com as informações, é o mais completo. Entretanto, os relatos também apontam algumas necessidades de funcionalidades que seriam úteis para uma melhor e mais efetiva aplicação no ambiente educacional.

\subsection{Discussão dos Resultados}

Os dados obtidos com esse trabalho demonstram, de forma simples, como o uso de tecnologias de comunicação e informação podem ser úteis no processo de ensino a surdos. Os 
dados demonstram que, dentre os aplicativos tradutores para língua de sinais estudados, o Hand Talk é o aplicativo que, além de entregar o maior número de funcionalidades, entrega praticamente todas, com exceção de uma, com a categoria A, além de ser o melhor avaliado pelos usuários e o mais utilizado pelos profissionais entrevistados. O Hand Talk também foi considerado pela ONU o melhor aplicativo social do mundo [Terenzi 2017]. Com base nos dados, foi observado a efetividade do Hand Talk e o seu potencial, sendo o aplicativo mais promissor. Apesar disso, o Hand Talk ainda não consegue atender todas as necessidades do ensino a surdos, como a necessidade de captura tradução de textos do mundo real. Sendo essa uma relevante necessidade, citada, inclusive, por um dos profissionais entrevistados e resolvida com o uso do Libras Lens. Portanto, mesmo sendo o mais promissor, o Hand Talk ainda precisa implementar muitas outras funcionalidades importantes para que consiga atender de forma satisfatória as necessidades presentes no processo educacional de surdos. Os outros aplicativos, por conseguinte, também possuem sua relevância dentro do processo de educação a surdos, cada um atuando dentro dos limites e efetividade de suas funcionalidades.

Os relatos dos profissionais, as avaliações de funcionalidades e as avaliações dos usuários dos aplicativos permitiram notar que, apesar de funcionais, os aplicativos ainda possuem muitos pontos que precisam ser evoluídos, atualizados e funcionalidades que precisam ser inseridas para que consigam alcançar o ponto ideal, mitigando os problemas de transcrição da língua oral e escrita para a língua de sinais e melhorando a comunicação e o entendimento dos surdos. Ainda assim, tais ferramentas são atraentes soluções para o ensino a surdos, se comparada a outros métodos, como as imagens estáticas, comumente utilizadas no ensino a surdos [Corrêa et al. 2014b] e [Vieira et al. 2014].

\section{Considerações Finais}

A partir das análises realizadas sobre aplicativos tradutores para LIBRAS, foi possível perceber o potencial que as ferramentas tecnológicas de informação e comunicação possuem para melhorar o processo de educação para os surdos. Ao analisar suas funcionalidades e compará-las com as experiências dos usuários, usando também relatos de as experiências dos profissionais do ensino a surdos, foi demonstrada a relevância dos tradutores para LIBRAS dentro do processo de ensino a surdos. Além de entender como os aplicativos facilitam na integração entre surdos e ouvintes e no desenvolvimento pessoal deles. Apesar de não possuir um caráter definitivo, esse artigo demonstra o impacto positivo dessas ferramentas dentro do processo de ensino a surdos e as características que podem ser melhoradas para que elas alcancem um nível maior de efetividade, uma vez que ainda não há uma grande quantidade quantidade de ferramentas adequadas disponibilizadas para a educação dos surdos [de Matos Lins 2011], sendo esse um estudo valioso.

De uma forma simples, este estudo demonstrou, também, que a maioria dos aplicativos avaliados possuem características e funcionalidades com potencial a ser explorado no ambiente educacional de surdos. A efetividade nas traduções, além de funcionalidades como a captura de textos do mundo real através da câmera do celular e o Regionalismo, com base nos dados, se mostraram bastante úteis. Além disso, um aplicativo de código aberto permite o estudo e desenvolvimento de novas funcionalidades ou novos aplicativos que podem auxiliar nesse cenário. Por fim, recomenda-se como proposta de continuidade da pesquisa a realização de experimentos de teste dessas aplicações em um ambiente real, além do estudo de suas funcionalidades e como incrementá-las, melhorá-las ou criar no- 
vas funcionalidades, como a tradução de LIBRAS para texto, não presente em nenhum dos aplicativos. Implicando isso, ou não, no desenvolvimento de uma ferramenta que atenda aos requisitos e necessidades da educação a surdos ou na atualização e correção das já existentes, auxiliando nas dificuldades encontradas no processo de ensino ao surdo e na inclusão do surdo, com o potencial para ser utilizado em qualquer situação.

\section{Referências}

Almeida, M. V. M., Alves, J. M., Jardim, J. J. d. S., Sales, E. R. d., et al. (2007). O ambiente logo como elemento facilitador na releitura de significados em uma atividade de ciências com alunos surdos. Anais do VII Encontro Latino-Americano de PósGraduação. São José dos Campos, UNIVAP.

Bandeira, I. D. C., Camillo, J. C., da Costa Neto, L. P., Muniz, P. B. V., da Purificação Pinto, R., and Guimarães, R. P. B. (2018). O uso do hand talk como meio de inclusão para alunos com deficiência auditiva na educação profissional. KiriKerê-Pesquisa em Ensino, (5).

Corrêa, Y., Gomes, R. P., and Ribeiro, V. G. (2017). Aplicativos de tradução portuguêslibras na educação bilíngue: desafios frente à desambiguação. RENOTE, 15(2).

Corrêa, Y., Gomes, R. P., and Ribeiro, V. G. (2018). Aplicativos de tradução portuguêslibras na educação bilíngue de surdos: tradução por meio de sinais ou datilologia? RENOTE, 16(1).

Corrêa, Y., Vieira, M. C., Santarosa, L. M. C., and Biasuz, M. C. V. (2014a). Aplicativos de tradução automática como facilitadores da comunicação entre surdos e ouvintes. Tecnologia e Acessibilidade, page 96.

Corrêa, Y., Vieira, M. C., Santarosa, L. M. C., and Biasuz, M. C. V. (2014b). Tecnologia assistiva: a inserção de aplicativos de tradução na promoção de uma melhor comunicação entre surdos e ouvintes. RENOTE, 12(1).

de Matos Lins, H. A. (2011). Alfabetização e letramento (também digitais) de alunos surdos: possibilidades de intervenção. Texto Livre: Linguagem e Tecnologia, 4(2):7481 .

Gil, A. C. (2008). Métodos e técnicas de pesquisa social. 6. ed. Editora Atlas SA.

Isquerdo, A. N. (1996). O fato linguístico como recorte da realidade sócio-cultural. 1996. PhD thesis, Tese (Doutorado em Linguística e Língua Portuguesa)-Universidade Estadual ....

Lima, W. D. (2017). Android e a influência do sistema operacional linux. Tecnologias em Projeção, 8(1):100-111.

Oliveira, J. P., Lopes, K. F., França, N. M., Santos, E. F., and Alvarenga, M. A. (2019). A utilização do aplicativo hand talk como ferramenta de apoio aos professores de ciências na educação inclusiva. Revista Interdisciplinar de Tecnologias e Educação, 5(1):14.

Pinto, M. A. d. S., Gomes, A. M. d. S., and Nicot, Y. E. (2012). A experiência visual como elemento facilitador na educação em ciências para alunos surdos. Revista Amazônica de Ensino de Ciências. 
Rodrigues, R. L., Maciel, A. M. A., and de Barros Carvalho Filho, E. C. (2012). Desenvolvimento de uma ferramenta para a produção de mídias utilizando personagem animado com síntese de voz. In Brazilian Symposium on Computers in Education (Simpósio Brasileiro de Informática na Educação-SBIE), volume 23.

Silva, J. F. S. d. (2021). Uma análise comparativa entre os aplicativos de tradução da língua portuguesa para a libras hand talk e vlibras. Dissertação (Especialista em LIBRAS), Instituto Federal de Ciência e Tecnologia da Paraíba.

Terenzi, D. (2017). Português-libras: Análise das traduções de verbos polissêmicos por tradutores automáticos. Entrelinhas, 11(2):211-222.

Vieira, M., Corrêa, Y., Santarosa, L., and Biazus, M. (2014). Além da interação: tradutores automáticos para língua de sinais e a constituição do sujeito surdo. International Society for Augmentative and Alternative Communication ISAAC. 